\title{
Nylon Dxygen Barrier Tubing Reduces Biofouling in Beer Draught Lines
}

\section{Parker Heger and Andrew R. Russell ${ }^{*}$}

Department of Biology

Northern State University

Aberdeen, SD 57401

Manuscript received 1 February, 2021; accepted 4 August 2021

*Corresponding author: Andrew.russell@northern.edu

Keywords: biofilm; biofouling; beer spoilage; nylon oxygen barrier; beer draught line; beverage tubing 


\section{Abstract}

Beer draught lines are frequently contaminated with biofilm-forming microorganisms, which forces retailers to spend considerable time and money cleaning and replacing lines. In light of this financial burden, draught tubing composition was examined for its role in the prevention of biofouling in beer lines. Three types of draught tubing - vinyl, polyethylene, and nylon barrier - were inoculated with a combination of biofilm-forming microorganisms (Hafnia paralvei, Raoultella planticola, Pediococcus damnosus and Saccharomyces cerevisiae) and used to simulate a bar environment for sixteen weeks. Following simulation, the degree of biofouling in each draught line was determined by spectrophotometry and microscopy. Absorption values and fluorescence images showed that nylon barrier tubing was superior to the other lines at resisting biofilm maturation.These results suggest that tubing composition plays a significant role in the prevention of biofilm formation in beer draught lines and supports the adoption of nylon barrier tubing as an effective strategy against biofouling in a variety of applications. 


\section{Introduction}

Biofouling creates a considerable financial burden at various levels of the beer industry. Arguably, the most challenging environment to keep clean is found in beer draught lines where yeast and spoilage organisms readily adhere, altering the taste, aroma, and quality of the beer. Although the deleterious effects of microbial biofilms have been recognized for several decades, relatively little scientific research has been applied to combat these issues, and most methods focus on routine maintenance of beer draught lines. For example, the Brewers Association recommends cleaning draught lines, at a minimum, every two weeks. The cleaning solution should be recirculated through the line for at least 15 minutes at a velocity of two gallons per minute. Additionally, all draught lines should be replaced annually as, despite regular and consistent cleaning, biofilms can still form (1).

Beer spoilage can be caused by a variety of microbial species. Perhaps the most notorious beer contaminants are lactic acid bacteria such as Lactobacillus brevis, and Pediococcus damnosus. These species are able to survive the harsh conditions of the brewing environment due to the presence of genes for hop resistance and polysaccharide production $(2 ; 25)$. While the biofilm formation of most lactic acid bacteria is relatively weak, their persistence and prevalence is enhanced by the presence of ubiquitous environmental species that have also been isolated from brewing equipment (14). Many isolates, such as Stenotrophomonas maltophilia, Pseudomonas putida, and Citrobacteri freundii, are known to be prolific biofilm producers and may aid lactic acid bacteria as secondary colonizers $(11 ; 24 ; 28)$. P. damnosus was recently isolated in our lab as a component of a multispecies biofilm in beer draught tubing from a local brewery (Russell et al, unpublished). Notably, a human commensal organism, Halfnia paralvei, a common soil inhabitant, Raoultella planticola, and brewer's yeast, Saccharomyces cerevisiae were the other predominant species in this biofilm.

As many retailers and distributers know, standard draught line cleaning procedures are very time-consuming, expensive, and only moderately effective (20). Although proper maintenance can delay biofilm growth and formation, cleaning of beer draught lines needs to be done consistently, as biofilms can form quickly and are more difficult to remove when mature due to the vast and multi-faceted defense mechanisms they exhibit (5). Consequently, alternative treatments have been developed for beer draught lines and other industrial applications that utilize enzymatic digestion $(10 ; 16 ; 29 ; 31)$. These approaches are moderately effective; however, they are often marketed as an additional step to a routine maintenance schedule, adding unnecessary time and expense.

Recently, the medical and industrial fields have shifted their focus to proactive methods of biofilm prevention rather than reactive treatment options. For example, a number of natural and artificial chemicals have been shown to possess antibiofilm properties that block quorum sensing (9), disperse extracellular polysaccharide (19), inhibit curli biosynthesis (6), or alter membrane permeability (13). A variety of studies also have been published in recent years describing options for making surfaces more resistant to biofouling, including development of novel materials $(17 ; 18)$, improvement of manufacturing methods $(15 ; 32)$, and creation of postproduction coatings $(7 ; 8 ; 22)$. Likewise, beer-draught line manufacturers have begun to experiment with various materials and manufacturing procedures to create lines that are more resistant to biofilm formation and, consequently, require less routine maintenance. One such product, known as Gen-X (Valpar), utilizes both a novel manufacturing method and addition of a nylon barrier layer to stop oxygen permeation and preserve beverage characteristics. Consequently, Gen-X tubing promises to ensure draught quality and hinder microbial growth 2-3 times more effectively than other alternatives (30). While this and other new proprietary compositions show promise at combatting biofouling, no peer-reviewed research has been done to characterize the effectiveness of these lines.

Our project aims to test the qualitative and quantitative effect that various tubing compositions have on biofilm formation in beer draught lines. We hypothesized that nylon barrier tubing would exhibit reduced biofouling compared to other tubing materials due to its potential to reduce oxygen permeation. To test this, three beer draught lines-vinyl, polyethylene, and nylon barrier (Gen-X)—were inoculated with a microbial cocktail consisting of Hafnia paralvei, 
Raoultella planticola, Pediococcus damnosus, and Saccharomyces cerevisiae liquid cultures. Following inoculation, the lines were connected to a keg-draught system from which beer was regularly dispensed for sixteen weeks to simulate a bar environment. Next, spectrophotometry and microscopy were used to determine the ability of the different draught tubing compositions to resist biofouling. Results from our experiments consistently showed that nylon barrier tubing was significantly better at resisting biofilm growth than traditional vinyl or polyethylene lines. The nylon barrier draught line showed impressive resistance to biofouling after the sixteen-week simulation, supporting the use of nylon oxygen barriers as a preventative measure against beer- spoilage biofilms.

\section{Methods}

\section{Microbial Cultures}

Four microbial species were obtained and cultured for this study as follows. Hafnia paralvei (ATCC 29927) and Raoultella planticola (ATCC 33431) were streaked onto nutrient agar plates and incubated at $37^{\circ} \mathrm{C}$. Pediococcus damnosus (ATCC 29358) was cultured on Lactobacilli MRS plates and incubated at $25^{\circ} \mathrm{C}$. Saccharomyces cerevisiae (Wyeast 1728) was streaked onto a malt agar plate and incubated at $25^{\circ} \mathrm{C}$. Individual $500 \mathrm{~mL}$ cultures of each species were inoculated with a single colony from each streak plate and incubated at the appropriate temperature until cultures reached late exponential phase ( -24 hours). Following incubation, all four cultures were mixed in a 1:1:1:1 ratio at OD600 -4.8 to create a microbial cocktail for inoculation of our beer draught lines.

\section{Simulated Dispensary System}

To simulate a standard dispensary system, three types of beer-draught lines, vinyl (Micromatic 550C), polyethylene (Micromatic 550NE), and nylon barrier (Micromatic 550BF), were attached to a single keg-containing an amber ale from Dakota Territory Brewing company, Aberdeen, $\mathrm{SD}$-and kept at $4^{\circ} \mathrm{C}$. Before being connected to the keg, all lines were cleaned with alkaline liquid beer line cleaner (Micromatic MM-B68), inoculated with a biofilm-forming microbial cocktail (described above), and left to incubate at room temperature for one hour. After inoculation, the culture was drained, and the lines were connected to the same keg using line splitters; the lines were then filled with beer. Once connected, $500 \mathrm{~mL}$ of beer was drawn from each line everyother day for sixteen weeks to simulate a bar environment, and kegs were replaced as needed. After the sixteen-week simulation, the lines were disconnected from the keg, wrapped in parafilm, and stored at $4^{\circ} \mathrm{C}$ for further processing; some beer was left in the line to prevent the line from drying while in storage.

\section{Quantifying Biofilm Formation}

To quantify biofilm formation in each tube, $25 \mathrm{~mm}$ long segments were cut at various regions from each line using a completely randomized design. Two segments were cut from each end and two segments were cut from the middle for a total of six samples in each treatment group.The samples were, then, rinsed lightly with deionized (DI) water, and one end was sealed with parafilm. Next, the samples were filled with $0.1 \%$ crystal violet and allowed to incubate for 10 mins. After incubation, the crystal violet was removed, and the samples were rinsed with DI water a second time to remove any unbound dye. Next, the tubes were filled with $1 \mathrm{~mL}$ of dimethylsulfoxide (DMSO) and incubated for 10 minutes. A stainless-steel spatula was used to gently agitate and break uplarger chunks of biofilm.

The sample was diluted 1:1 with more DMSO, loaded into a $2 \mathrm{~mL}$ cuvette, and the absorbance was measured at $\lambda 600$ on a spectrophotometer. Results were analysed using a one factor ANOVA to assign statistical significance.

\section{Fluorescence Microscopy}

One foot of tubing was taken from each type of beer line and three $12.5 \mathrm{~mm}$ samples were cut from each foot-long segment. The samples were then cut longitudinally into semicircles and stained using the LIVE/DEAD BacLight bacterial viability kit (Invitrogen), according to the manufacturer's recommendations. The sections were then attached to a slide using tape and observed with fluorescence microscopy. Images 
were acquired using a semi-randomized, single blind method on a Leica DM8 confocal microscope with an excitation wavelength of $480 \mathrm{~nm}$ and a 63x oil immersion objective.

\section{Results}

\section{Quantifying Biofilm Formation}

After disassembling our simulated draught system, each draught line was stained with crystal violet to compare total biofilm formation between the three tube types.Visual inspection of each line showed a dramatic difference between the nylon barrier tubing and the other two types. Some biofouling was evident in the nylon barrier tubing, but it was visibly cleaner than both the polyethylene and the vinyl lines (Fig. 1). To quantify this difference, each tube was stained with crystal violet and the absorbance was measured using a spectrophotometer. Matching its visible appearance, the vinyl beer draught line had the highest average absorbance value at 2.771 with a standard deviation of $\pm 0.464(2.771 \pm$ $0.464, \mathrm{n}=3$ ). Biofilm from the polyethylene line measured an absorbance value of 1.601 with a standard deviation of $\pm 0.447(1.601 \pm 0.447, n=3)$. Notably, the nylon barrier line exhibited significantly lower absorbance than both the vinyl $(\mathrm{p}=0.00001)$ and the polyethylene $(\mathrm{p}=0.0004)$ lines, measuring an average value of 0.253 with a standard deviation of $0.139(0.253 \pm 0.139, n=3)$ (Fig. 2). It should be noted that crystal violet stain does not differentiate between viable and dead cells in this assay. Therefore, the mean absorbance values reported herein are a measurement of cells that are alive and those that are not.

\section{Fluorescence Microscopy}

While the crystal violet staining of the nylon barrier tubing was significantly different from the other two materials, these results gave little insight into the biofilm characteristics and microarchitecture on each tube type. In particular, they did not indicate if bacteria and yeast were prevented from adhering to the nylon barrier tubing altogether, or if microbial cells were just concentrated in small, localized biofilms dispersed along its surface. Therefore, confocal fluorescence microscopy was utilized to obtain three-dimensional images of the biofilms on each tube type. After removing three 25 $\mathrm{mm}$ segments from each line, the cells were stained with a fluorescent dye and observed by confocal microscopy to view the characteristics of biofilms in each microenvironment. Image analysis revealed that most of the inner surface on the vinyl line was covered with a thin, but mature biofilm (Fig. 3, left column). By comparison, the polyethylene line exhibited substantially less biofouling, but still had a moderate level
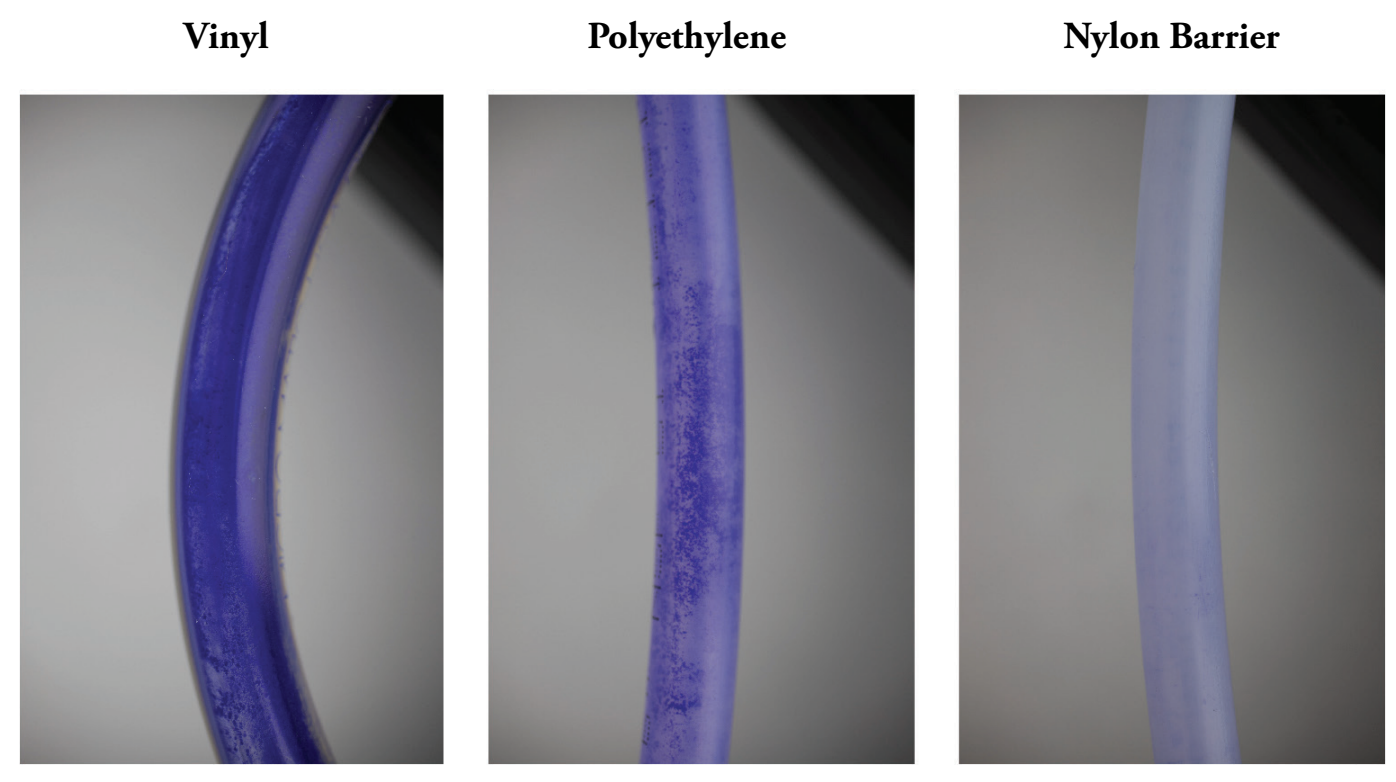

Figure 1. Crystal violet staining of draught lines. Following a sixteen-week simulation, each draught line was drained, rinsed with DI water, and stained with crystal violet. Qualitative images were taken to compare relative biofouling. 
of bacteria adhered to its surface (Fig. 3, middle column). Similar to the polyethylene line, the nylon barrier tubing exhibited considerable resistance to biofouling. Only single cells were adhered to its surface, with no evidence of a cohesive biofilm (Fig. 3, right column).

\section{Discussion}

The maintenance of beer draught lines is an expensive and time-consuming process that affects thousands of businesses around the globe. Standard line maintenance is essential to ensure taste, aroma, and quality of the product, but it involves cleansing of the line using acidic or alkaline chemicals at a minimum every 2-3 weeks and complete line replacement yearly. Improper cleaning of beer draught lines allows for microorganisms to adhere in the lines while feeding off the nutrients provided by the beer. The adhered microorganisms multiply in the line, and once there are sufficient organisms in the surrounding environment, the organisms begin to produce a polysaccharide matrix around themselves for protection. This biofilm makes the beer-spoiling organisms difficult to eradicate,costing retailers and distributors money and time. For these reasons, the prevention of biofilm formation is of great importance.

In recent years, tubing manufacturers have been developing new chemical compositions that promise increased resistance to microbial biofilms and, therefore, longer intervals between line cleanings. To our knowledge, our results represent the first peer-reviewed study to support the claims that nylon barrier tubing with reduced oxygen permeability does, indeed, resist biofouling. Our findings indicate that the nylon barrier line

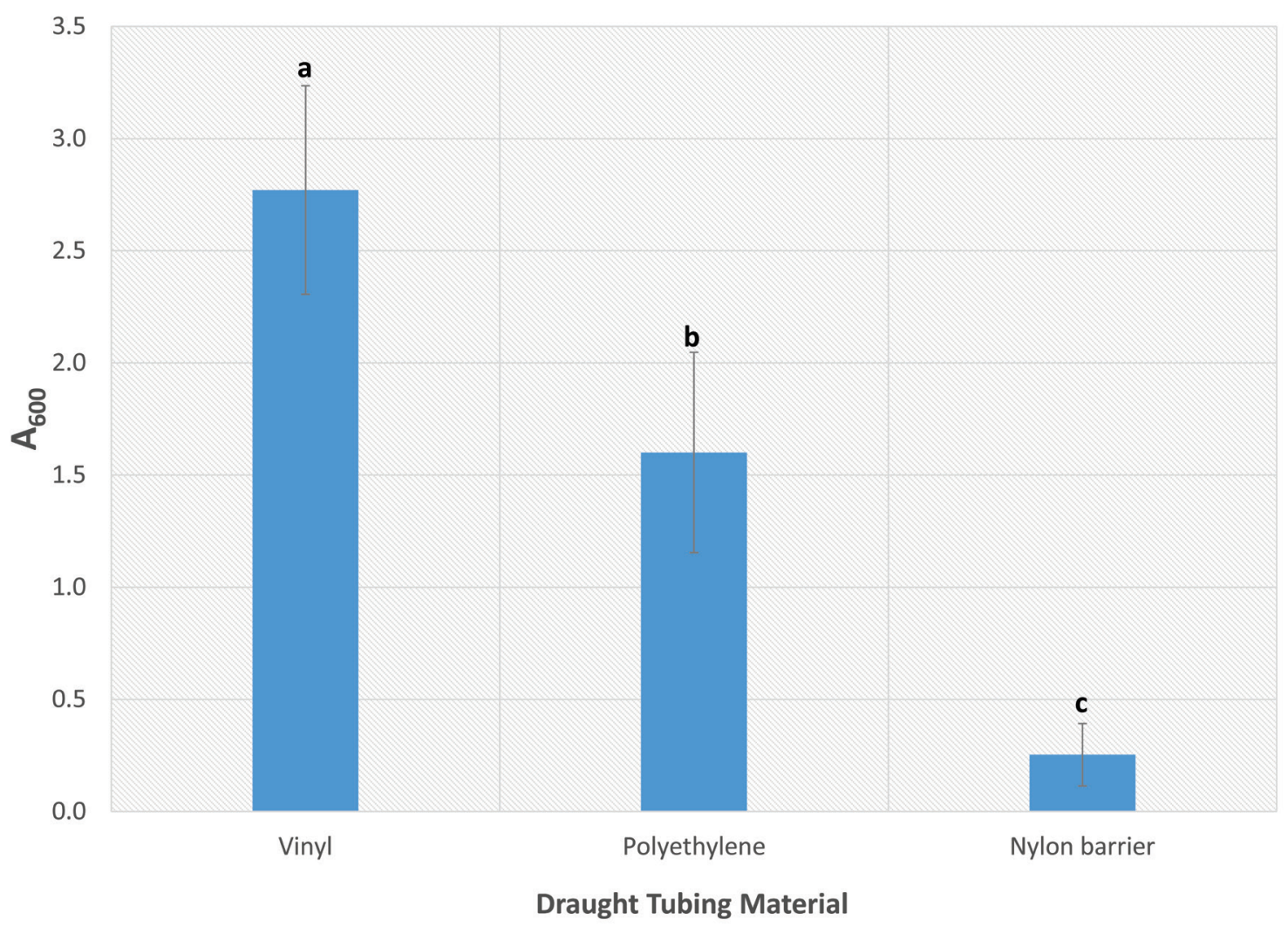

Figure 2. Quantifying biofouling of draught lines. Six $25 \mathrm{~mm}$ segments were taken from each draught line and stained with crystal violet. The stained biofilm was solubilized with DMSO, transferred to acuvette, and absorbance was measured at $\lambda 600$. Errorbarsrepresentstandarddeviation in each group. Significant differences wereobtained between groups indicated with different letters;

${ }^{*} \mathrm{P}<0.01$.

30 | Fine Focus 
is roughly six times more resistant to biofilm formation than polyethylene tubing and ten times better than standard vinyl tubing (Fig. 2). Fluorescence microscopy revealed that this is due to fewer cells adhering to the surface of the nylon barrier line compared to the others (Fig. 3). While moderate levels of microbial cells still adhere to the surface of both polyethylene and nylon barrier tubing, the microenvironments in these tubes do not promote biofilm maturation. It is likely that the microbial population density required to establish a biofilm has not been reached.

Although the results from this experiment show that the structural and chemical composition of beer line affects biofouling, there are several caveats that deserve consideration. First, it could be assumed that the nylon barrier line helps preserve the quality of the beer longer because it inhibits biofouling. However, the taste and quality of the beer was not evaluated as a part of this study. More research might be needed to substantiate the benefit to consumers. Second, only one style of beer - an amber ale - was used in this study; results may vary with beer styles containing higher alcohol content, greater alpha-acid levels, differing $\mathrm{pH}$, etc. Research suggests that each beer style may provide a unique growth environment, supplying specific micronutrients and antimicrobial compounds (21). For example, hops content is known to have a significant effect on the types of microorganisms that survive in different beer styles $(23 ; 26$; 27). Knowing these differences could be useful to optimize cleaning protocols for beer draught lines dedicated to certain beer styles. Third, each line was directly inoculated with a very high concentration (OD600 4.8) of microorganisms and those cells were allowed to establish themselves in the absence of a regular cleaning regimen for sixteen weeks. These conditions were chosen to ensure adequate adherence of primary biofilm colonizers and to accentuate the potential impact of each tubing material. These conditions may not

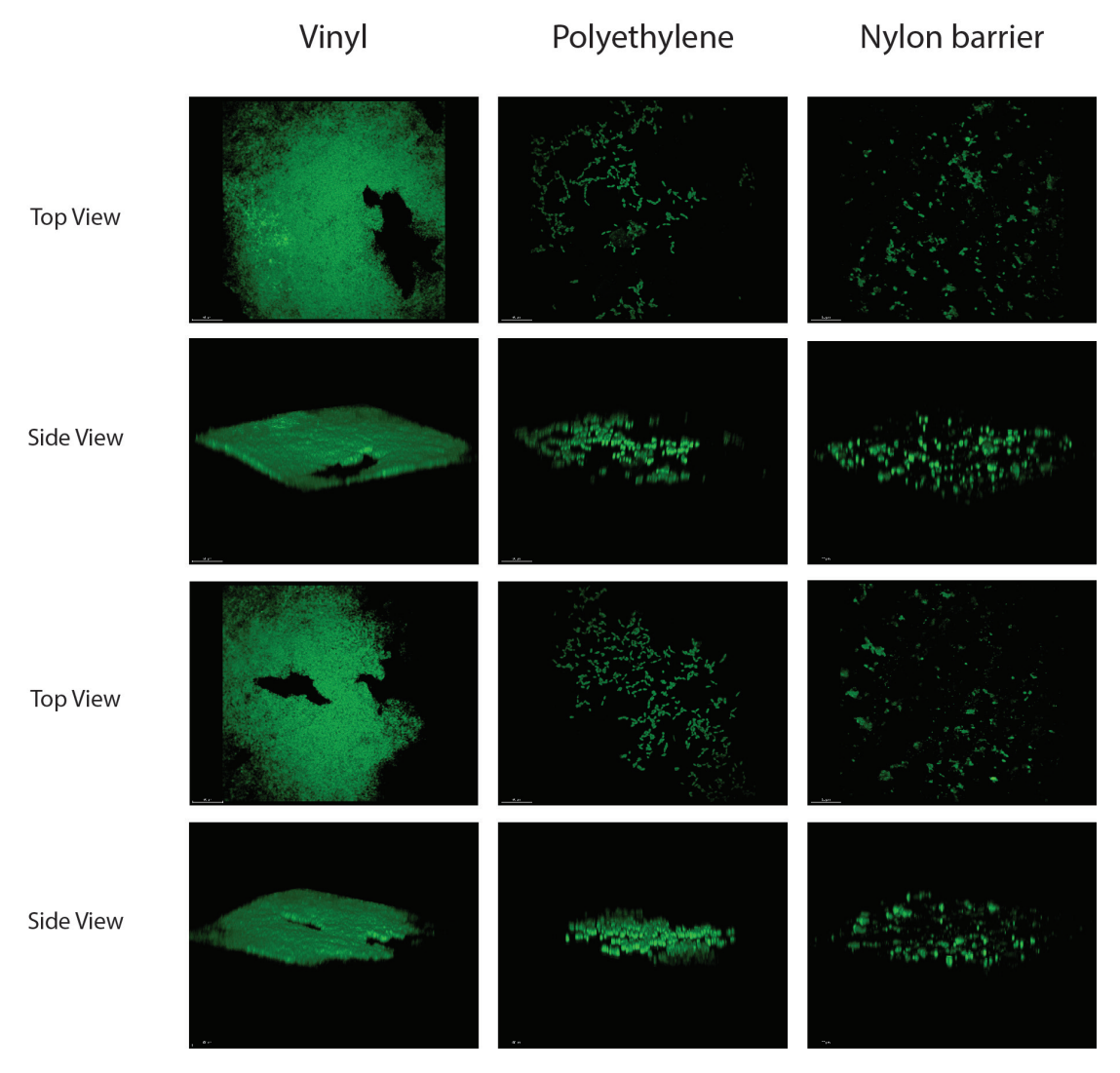

Fig. 3. Confocal imaging of adhered biofilm. Six $25 \mathrm{~mm}$ segments were taken from each draughtline, stained with a fluorescent dye, and observed on a confocal microscope with an excitation wavelength of $480 \mathrm{~nm}$ and a $63 \mathrm{x}$ oil immersion objective. 
reflect the typical biological conditions that most beer retailers and distributors encounter. Finally, there are many anaerobic organisms such as Megasphaera and Pectinatus species that are known to adhere to brewery surfaces and cause beer spoilage $(3 ; 4)$. Since all four of the test organisms in our study were aerobic or facultative anaerobes, our results cannot predict the potential impact that nylon barrier tubing might have on biofilms formed by strict anaerobes. Given that the benefit of nylon barrier tubing is due to a presumed reduction in oxygen permeability (12), it is likely that lines containing this barrier would show little to no impact on the growth of anaerobic microorganisms.

Despite the caveats described above, our study provides convincing evidence that nylon barrier tubing substantially reduces biofouling when used for beer dispensing. The adoption of such tubing is a low-cost option for retailers to improve the quality of their product and reduce the frequency of line cleaning, resulting in less long-term expense. Moreover, the use of such nylon barriers could have wideranging benefits in other applications such as water and soft drink lines, diagnostic equipment, and medical devices.

\section{Acknowledgments:}

We would like to thank Brodie Mueller and DakotaTerritory Brewing Company for providing used draught lines, equipment for the simulated draught system, and the ale for experimentation. We would also like to acknowledge Dr. Darla Goeres at Montana State University for her advice on experimental design.

\section{Disclosure Statments:}

The authors declare no conflict of interest.

\section{Funding:}

This work was supported by the National Science Foundation EPSCoR Research Infrastructure Improvement Program under the RII Track-1 Grant \# 1849206.

32 | Fine Focus 


\section{References}

1. A1. Association, B. (2014). Brewers Association Draught Beer Quality for Retailers. Retrieved from https://www. brewersassociation.org/educational-publications/draught-beer-quality-for-retailers/

2. Bergsveinson, J., Baecker, N., Pittet, V., \& Ziola, B. (2015). Role of Plasmids in Lactobacillus brevis BSO 464 HopTolerance and Beer Spoilage. Appl Environ Microbiol, 81(4), 1234-1241. doi:10.1128/ Aem.02870-14Arnesen, L., P. Stenfors, A. Fagerlund, and P.E. Granum. 2008. From soil to gut: Bacillus cereus and its food poisoning toxins. FEMS Microbiol. Rev. 32:579-606.

3. Bittner, M., de Souza, A. C., Brozova, M., Matoulkova, D., Dias, D. R., \& Branyik, T. (2016). Adhesion ofanaerobicbeerspoilagebacteriaMegasphaeracerevisiaeandPectinatusfrisingensistostainlesssteel. Lwt-Food Science and Technology, 70, 148-154. doi:10.1016/j.lwt.2016.02.044

4. Bittner, M., Strejc, J., Matoulkova, D., Kolska, Z., Pustelnikova, L., \& Branyik, T. (2017). Adhesion of Megasphaeracerevisiaeontosolidsurfacesmimickingmaterialsusedinbreweries.JournaloftheInstitute of Brewing, 123(2), 204-210. doi:10.1002/jib.415

5. Bridier, A., Briandet, R.,Thomas,V.,\& Dubois-Brissonnet, F. (2011). Resistanceofbacterialbiofilmsto disinfectants: a review. Biofouling, 27(9), 1017-1032. doi:10.1080/08927014.2011.626899

6. Cegelski, L., Pinkner, J. S., Hammer, N. D., Cusumano, C. K., Hung, C. S., Chorell, E., . . Hultgren, S. J.(2009). Small-moleculeinhibitorstargetEscherichiacoliamyloidbiogenesisandbiofilmformation. Nat Chem Biol, 5(12), 913-919. doi:10.1038/nchembio. 242

7. Dundas, A. A., O. Sanni, J. F. Dubern, G. Dimitrakis, A. L. Hook, D. J. Irvine, P. Williams, and M. R. Alexander. "Validating a Predictive Structure-Property Relationship by Discovery of Novel Polymers Which Reduce Bacterial Biofilm Formation." [In English]. Advanced Materials 31, no. 49 (Dec 2019).

8. R. (2019). Validating a Predictive Structure-Property Relationship by Discovery of Novel Polymers whichReduceBacterialBiofilmFormation.AdvancedMaterials,31(49).doi:ARTN190351310.1002/ adma.201903513

9. Fisher, L., Ostovapour, S., Kelly, P., Whitehead, K. A., Cooke, K., Storgards, E., \& Verran, J. (2014). Molybdenumdopedtitaniumdioxidephotocatalyticcoatingsforuseashygienicsurfaces:theeffectof soiling on antimicrobial activity. Biofouling, 30(8), 911-919. doi:10.1080/08927014.2014.939959

10. Hentzer, M., Riedel, K., Rasmussen, T. B., Heydorn, A., Andersen, J. B., Parsek, M. R., . . . Givskov, M. (2002). InhibitionofquorumsensinginPseudomonasaeruginosabiofilmbacteriabyahalogenated furanone compound. Microbiology-Sgm, 148, 87-102. doi:Doi 10.1099/00221287-148-1-87

11. Itoh, Y., Wang, X., Hinnebusch, B. J., Preston, J. F., \& Romeo, T. (2005). Depolymerization of beta-1,6- N-acetyl-D-gluc osaminedisruptstheintegrityofdiversebacterialbiofilms.JBacteriol,187(1),382-387. doi:10.1128/Jb.187.1.382-387.2005

12. Jucker, B. A., Harms, H., \& Zehnder, A. J. (1996). Adhesion of the positively charged bacterium Stenotrophomonas (Xanthomonas) maltophilia 70401toglassandTeflon.JBacteriol,178(18),5472- 5479. doi:10.1128/jb.178.18.54725479.1996 
13. Khanna, Y. P., Day, E. D., Tsai, M. L., \& Vaidyanathan, G. (1997). Re-examining the oxygen barrier of nylon6films.1.Roleofmoistureandprocessinginducedvariables.JournalofPlasticFilm\&Sheeting, 13(3), 197-211. doi:Doi $10.1177 / 875608799701300304$

14. Kharidia, R., \& Liang, J. F. (2011). The Activity of a Small Lytic Peptide PTP-7 on Staphylococcus aureus Biofilms. Journal of Microbiology, 49(4), 663-668. doi:10.1007/s12275-011-1013-5

15. Maifreni, M., Frigo, F., Bartolomeoli, I., Buiatti, S., Picon, S., \& Marino, M. (2015). Bacterial biofilm asapossiblesourceofcontaminationinthemicrobreweryenvironment.FoodControl,50,809-814. doi:10.1016/j. foodcont.2014.10.032

16. Narayana, P. S. V. V. S., \& Srihari, P. S. V. V. (2019). Biofilm Resistant Surfaces and Coatings on Implants: A Review. Materials Today-Proceedings, 18, 4847-4853.

17. Nijland, R., Hall, M. J., \& Burgess, J. G. (2010). Dispersal of Biofilms by Secreted, Matrix Degrading, Bacterial DNase. PLoS One, 5(12). doi:ARTN e1566810.1371/journal.pone.0015668

18. Park, H. H., Sun, K., Lee, D., Seong, M., Cha, C., \& Jeong, H. E. (2019). Cellulose acetate nanoneedle arraycoveredwithphosphorylcholinemoietyasabiocompatibleandsustainableantifoulingmaterial. Cellulose, 26(16), 8775-8788. doi:10.1007/s10570-019-02681-w

19. Park, H. H., Sun, K., Seong, M., Kang, M., Park, S., Hong, S., . . Jeong, H. E. (2019). Lipid-Hydrogel- NanostructureH ybridsasRobustBiofilm-ResistantPolymericMaterials.AcsMacroLetters, 8(1),64-69. doi:10.1021/acsmacrolett.8b00888

20. Park, S. C., Park, Y., \& Hahm, K. S. (2011). The Role of Antimicrobial Peptides in Preventing Multidrug-Resistan tBacterialInfectionsandBiofilmFormation.InternationalJournalofMolecular Sciences, 12(9), 5971-5992. doi:10.3390/ ijms12095971

21. Quain,D.E.(2016).Draughtbeerhygiene:cleaningofdispensetapnozzles.JournaloftheInstituteof Brewing, 122(3), 388396. doi:10.1002/jib.335

22. Riedl, R., Goderbauer, P., Brandl, A., Jacob, F., \& Hutzler, M. (2017). Bavarian Wheat Beer, an Example ofaSpecialMicrobeHabitat-Cultivation,Detection,BiofilmFormation,CharacterizationofSelected Lactic Acid Bacteria Hygiene Indicators and Spoilers. Brewing Science, 70(1-2), 39-50.

23. Sae-ung, P., Wijitamornloet, A., Iwasaki, Y., Thanyasrisung, P., \& Hoven, V. P. (2019). Clickable Zwitterionic Copolymer as a Universal Biofilm-Resistant Coating. Macromolecular Materials and Engineering, 304(9). doi:ARTN 190028610.1002/mame.201900286 
24. Sami, M., Suzuki, K., Sakamoto, K., Kadokura, H., Kitamoto, K., \& Yoda, K. (1998). A plasmid pRH45ofLactobacillusbrevisconfershopresistance.JournalofGeneralandAppliedMicrobiology, 44(5), 361-363. doi:DOI $10.2323 /$ jgam.44.361

25. Shrove, G. S., Olsen, R. H., \& Vogel, T. M. (1991). Development of pure culture biofilms of $P$. putida on solid supports. Biotechnol Bioeng, 37(6), 512-518. doi:10.1002/bit.260370604

26. Snauwaert, I., Stragier, P., De Vuyst, L., \& Vandamme, P. (2015). Comparative genome analysis of Pediococcus damnosus LMG28219, astrainwell-adaptedtothebeerenvironment.BMCGenomics, 16, 267. doi:10.1186/s12864-015-1438-z

27. Suzuki, K., Iijima, K., Ozaki, K., \& Yamashita, H. (2005). Isolation of a hop-sensitive variant of Lactobacillus lindneri and identification of genetic markers for beer spoilage ability of lactic acid bacteria. Appl Environ Microbiol, 71(9), 5089-5097. doi:10.1128/Aem.71.9.5089-5097.2005

28. Suzuki, K., Iijima, K., Sakamoto, K., Sami, M., \& Yamashita, H. (2006). A review of hop resistance in beer spoilage lactic acid bacteria. Journal of the Institute of Brewing, 112(2), 173-191. doi:DOI 10.1002/j.2050-0416.2006.tb00247.x

29. Thompson, L. J., Gray, V., Lindsay, D., \& von Holy, A. (2006). Carbon : nitrogen : phosphorus ratios influence biofilm formation by Enterobacter cloacae and Citrobacter freundii. Journal of Applied Microbiology, 101(5), 1105-1113. doi:10.1111/j.1365-2672.2006.03003.x

30. Tsiaprazi-Stamou, A., Monfort, I. Y., Romani, A. M., Bakalis, S., \& Gkatzionis, K. (2019). The synergisticeffectofenzymaticdetergentsonbiofilmcleaningfromdifferentsurfaces.Biofouling,35(8), 883-899. doi:10.1080 /08927014.2019.1666108

31. Valpar Beverage Tubing. (2000-2019, 2019). Retrieved from https://www.pasp.com.br/valpar- beverage-tubing.html

32. Walker, S. L., Fourgialakis, M., Cerezo, B., \& Livens, S. (2007). Removal of microbial biofilms from dispenseequipme nt:Theeffectofenzymaticpre-digestionanddetergenttreatment.Journalofthe Institute of Brewing, 113(1), 61-66. doi:DOI 10.1002/j.2050-0416.2007.tb00257.x

33. Zhou, C., Song, H. Q., Loh, J. L. C., She, J. Q., Deng, L. H., \& Liu, B. (2018). Grafting antibiofilm polymer hydrogel film onto catheter by SARA SI-ATRP. Journal of Biomaterials Science-Polymer Edition, 29(17), 2106-2123. doi:10.10 80/09205063.2018.1507268 